\title{
Color Face Recognition Using Quaternion PCA
}

\author{
Emad S. Jaha ${ }^{1}$ and Lahouari Ghouti ${ }^{2}$ \\ ${ }^{1}$ King Abdulaziz University. Jeddah. Saudi Arabia. \\ ${ }^{2}$ King Fahd University of Petroleum and Minerals. Dhahran 31261. Saudi Arabia.
}

Keywords: Biometric Systems, Face Recognition, Color Face Recognition, Principal Component Analysis (PCA), Hypercomplex PCA.

\begin{abstract}
Recently, biometric systems have attracted the attention of both academic and industrial communities. Advances in hardware and software technologies have paved the way to such growing interest. Nowadays, efficient and cost-effective biometric solutions are continuously emerging. Fingerprint-based biometric systems have emerged as pioneering commercial applications of biometric systems. Face and iris traits have proven to be reliable candidates. Until recently, face recognition research literally followed the research undertaken in the field of fingerprint recognition which is inherently gray-scale. In this paper, efforts are restricted to the investigation of face representations in the color domain. The concept of principal component analysis (PCA) is carried over into the hypercomplex domain (i.e., quaternionic) to define quaternionic PCA (Q-PCA) where color faces are compactly represented. Unlike the existing approaches for handling the color information, the proposed algorithm implicitly accounts for the correlation that exists between the face color components (i.e., red, green and blue, respectively).
\end{abstract}

\section{Introduction}

Recent advances in computer technology and electronic packaging have paved the way for the emergence of state-of-the-art access control and identity verification/authentication systems such as banking systems, border-access control and access control. Such system use different forms of technology. However, the commonly used solutions consist of username/password, electronic and smart cards. Although these solutions are effective and have many advantages, they suffer from serious uncontrollable disadvantages. Several violations could be easily performed such as using a stolen username/password or electronic/smart card by unauthorized persons. Another drawback consists in losing or forgetting the identity mean (i.e., username/password or smart card), which can also prevent the legal person from accessing the system. Also, other various forms of violations and problems can occur effortlessly. Due to such disadvantages, biometric solutions have taken place and started playing a main role in developing efficient authentication and identification systems which are free from the above mentioned drawbacks. Biometric-based systems make use of various unique human traits. The majority of earlier research efforts have focused on fingerprint-based biometric systems. After that, face and iris traits have become the focus of very active research. Face recognition (FR) has many advantages over other biometric traits because it is natural, nonintrusive, and easy to use. Face recognition research has literally followed fingerprint recognition research in methodologies and techniques used. Therefore, most of published research is mostly based on gray-scale face images. This approach has handicapped many of the existing FR algorithms to benefit from the rich information interaction that takes place implicitly in the color domain. This paper proposes a remedial solution to this drawback since the use of gray-scale representations would lead to the loss of valued color information. Needless to say that there are some attempts in the literature where each color component is used independently of the remaining color components. In this case, color independence is assumed which violates the basic principles of computer vision. Unlike existing color FR algorithms, in this paper, a solution based on hypercomplex (i.e., quaternionic) image representation is proposed where the color information is used in a "holistic" manner. Hence, the proposed solution implicitly accounts for the correlation that exists between the color components. Figure 1 depicts a categorization of FR methods where the proposed color technique is also illustrated. This paper is organized as follows. Section 1 provides an introduction to the color face recognition problem. Then, face recognition concepts and techniques along with a review of the current literature are laid out in Section 2. In Section 3 , an overview of quaternion (hypercomplex) concepts is provided where basic definitions, properties, and representations are discussed therein in details. A detailed description of the proposed quaternionic principal component analysis (Q-PCA) method is presented along with related mathematical expression of singular value decomposition (SVD) and its quaternionic counterpart (Q-SVD) in Section 4. Section 5 outlines the adopted methodology for the experiments conducted and computer simulation carried out in this paper. The outline is followed by a summary of the reported results and a detailed performance analysis. Finally, the paper concludes with Section 6 where the paper contributions are summarized and conclusions drawn. 


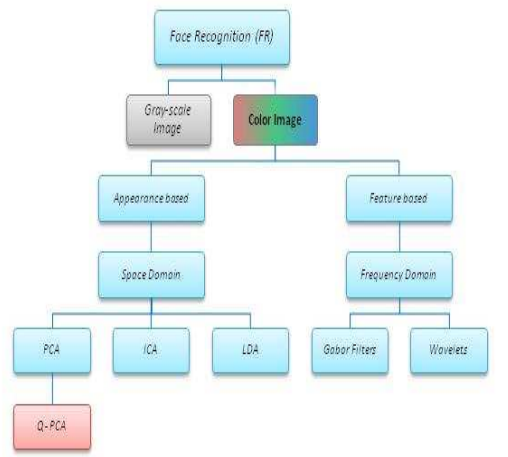

Figure 1. Categorization of face recognition (FR) methods.

\section{Review of Face Recognition Algorithms}

A face recognition (FR) system is simply an attempt to emulate the typical human face recognition task that a human performs routinely, effortlessly, and frequently along his life. Thus face recognition defined as the ability of a computer to receiving and interpreting of face image input. Such face recognition system is supposed to identify faces presented in images and videos automatically. It can operate in either a single or dual mode. Like other biometric systems, an FR system is capable of [1]:

1) Capturing a face sample from an end user.

2) Extracting biometric data from that sample.

3) Comparing the biometric data with that contained in one or more reference templates.

4) Deciding how well they match.

5) Indicating whether or not an identification or verification of identity has been achieved.

Face recognition has undergone approximately fifteen years of intensive development in both academic and commercial arenas[1]. Despite of there exist several different approaches in the academic literature, they can be divided into four major classes of algorithms:

1) Eigenface Systems based on eigenfaces and eigenvectors.

2) Local Feature Analysis System based on the analysis of local features.

3) Neural Network methods based on machine learning techniques.

4) Gabor filter methods based on Gabor and wavelets analogies.

Definitely, each class has its own advantages and disadvantages. The diagram, shown in Figure 1, demonstrates classification of FR methods. Although, FR is a very popular and promising research topic, the task is also tending to be a difficult one due to the existence of unconstrained (or uncontrolled) environments such as viewpoint, illumination, expression, occlusion, and accessories.

\subsection{Face Recognition Processing}

The face recognition processing flow, depicted in Figure 2, consists of four modules: detection, alignment, feature extraction, and matching. Generally speaking, the whole system modules or steps can be divided into two main stages: preprocessing and recognition stages. The former stage includes face detection and alignment or localization and normalization. Facial feature extraction and matching constitute the latter stage.

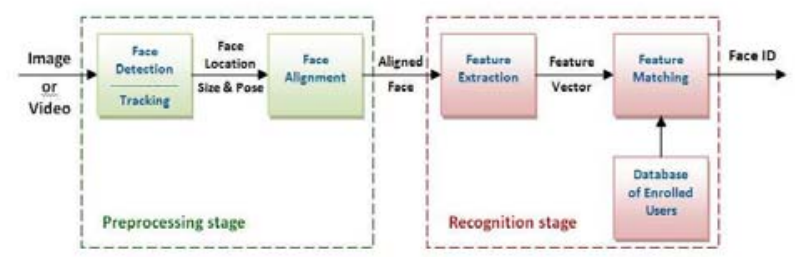

Figure 2. Face recognition processing flow.

Face detection segments the face areas from the background known as "nonface" segments. Face alignment is used to achieve more accurate localization, and at normalizing faces thereby it can treat the coarse estimates of the location and scale of each detected face which is detected and provided by face detection module. Figure 3 illustrates face detection and alignment processes. The two processes yield the outlines of located and normalized facial components outlines.

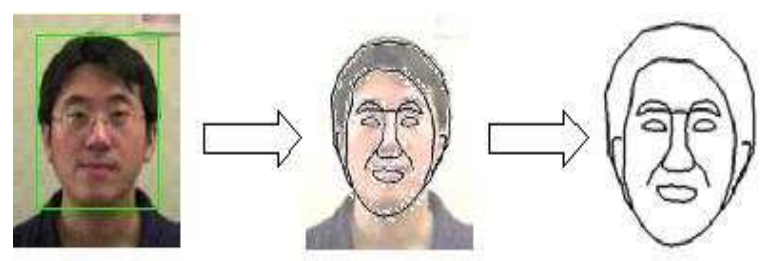

Figure 3. Face detection and alignment processes.

\subsection{Existing Color-based Face Recognition Algorithms}

It should be noted that several approaches that make use of color information have been proposed [2, 3]. Choi et al. [4] propose a metric called "variation ratio gain" (VRG) to theoretically prove the significance of color effect on low-resolution faces. In addition, extensive performance comparison studies are conducted. Yang and Liu [2] introduced a General Discriminant Model (GDM) for color face recognition. It is deals with two sets of variables: a set of color component combination coefficients for color image representation and a set of projection basis vectors for image discrimination. In order to find the optimal solution of the model an iterative whitening-maximization 
(IWM) algorithm is designed and used. The diagonal nonnegative matrix factorization (BDNMF) is proposed by Wang et al. [5] for color face representation and recognition. The approach employs block diagonal matrix to encode color information of different channels. An adapted Nearest Neighborhood classifier is used to identify color face samples. Whereas the obtained experimental results on (CVL) and (CMU PIE) color face databases verify the effectiveness of the proposed approach. Thomas et al. [6] investigate the use of the characteristics of a 3D color to generate a color Linear Discriminant Analysis (LDA) subspace, which could be used to recognize a new testing image. In order to test the potential improvement in accuracy, a recognition rate across two color face databases is computed. The observed results indicated that the LDA color subspace significantly improves recognition accuracy over the standard grayscale approach. Wang et al. [7] propose a color face recognition approach based on 2D-PCA. The proposed approach comprises a matrix-representation model that encodes the color information directly to describe the color face image. In this way, color face images are represented efficiently in the matrix format. Consequently, color-eigenfaces are computed for feature extraction using 2D-PCA, and the Nearest Neighborhood classification is adapted to identify the color face samples. Moon and Phillips [8] introduce a generic modular PCAalgorithm in order to investigate the influence of the computational and performance aspects of PCA-based for face recognition algorithms.

\subsection{Color Quaternion PCA (Q-PCA) Approach}

A technique for quaternion matrix algebra which can be used to process the eigen analysis of a color image is proposed by Le Bihan and Sangwine [9]. This technique introduces extensions of two classical techniques to their quaternionic case: Singular Value Decomposition (SVD) and Karhunen-Loeve Transform (KLT). It also introduces the problem of Eigne Value Decomposition (EVD) of quaternion matrix. The properties of these quaternion tools are given and their behavior on natural color images is presented. Furthermore, a method to compute the decomposition using complex matrix algebra is provided. In addition, another consequent work by Sangwine and Le Bihan [10], the Jacobi algorithm for computing the quaternion SVD (Q-SVD) is presented. Based on Q-SVD, Shi [11] implements the Q-PCA, and applies it to several applications such as color image segmentation. Trilateral filtering is proposed by locally adapting color and changing the shape of the filter to achieve the effect of smoothing colors that preserve the edges. Ding and Feng [12] propose another method of quaternion Karhunen-Loeve Transform (Q-KLT) and biomimetic pattern recognition (BPR) for color face recognition. The model of (Q-KLT) is used to extract the eigen-faces of training samples and algebraic features for each by BPR method. A method proposed by some researchers called Extended Two-Dimensional PCA (E2D-PCA) which is an extension to the original 2D-PCA for gray face recognition. E2D-PCA is promoted by a new covariance matrix preserving more local geometric structure information than previous 2D-PCA methods. It avoids small sample size problem of the PCA method. Although the new E2D-PCA improves the previous 2D-PCA, the former one still has some limitation that, it is only considering the global information of face images. Besides, it is only applied for gray face recognition. Where some local and color information may be ignored. Thus, Chen et al. [13] provide a solution to such problems by proposing SpE2D-PCA a hybrid approach based on sub-pattern technique and E2D-PCA.

\section{Quaternion (Hypercomplex) Representations}

\subsection{Quaternion Numbers}

Quaternions were first proposed by Hamilton in 1843 [10]. Quaternions are associative but non-commutative and they belong to a specific class of hypercomplex numbers. Such numbers are used in several applications such as mechanics in 3D space and 3D rotations [14]. They are also used in image processing to represent and process color images in a holistic manner rather than handling and processing each color component separately. Complex numbers represented as a combination of a real and an imaginary parts such as:

$$
c=a+b \cdot i
$$

where $a, b$, and $i$ represent the real, imaginary components and the imaginary unit. Quaternions can be considered as a generalization of complex numbers having three imaginary components [11]. A quaternion number can be written as a linear combination defined as follows:

$$
q=a+b \cdot i+c \cdot j+d \cdot k
$$

where the quaternion number $q$ consists of one real part, $a$, and three imaginary parts $(b, c$, and $d)$. The three imaginary units are represented by $i, j$ and $k$, respectively.

Moreover, the hypercomplex set, $\mathbb{Q}$, is related to the "regular" complex and real sets $(\mathbb{C}$ and $\mathbb{R})$ as follows:

$$
\mathbb{Q} \subseteq \mathbb{C} \subseteq \mathbb{R}
$$
erties:

The imaginary units $i, j$ and $k$ satisfy the following prop-

$$
i j k=i^{2}=j^{2}=k^{2}=-1
$$

$$
(i j=-j i=k),(j k=-k j=i),(k i==-i k=j)
$$

Other useful properties can be found in [11]. 


\subsection{Quaternion Representation of Color Face Images}

Each pixel in a color image has the property of having the values of the three color channels or components. These three colors simultaneously represent a pixel and are defined as the three imaginary parts of the quaternion. Thus, any pixel or point, $(x, y)$, of a color face image can be represented by a pure quaternion as follows:

$$
q_{x y}=0+R_{x y} \cdot i+G_{x y} \cdot j+B_{x y} \cdot k
$$

where $R_{x y}, G_{x y}$ and $B_{x y}$ represent the three color components at pixel $(x, y)$, respectively. Fig. 4 illustrates the approach adopted to represent RGB color face images using a quaternionic form.

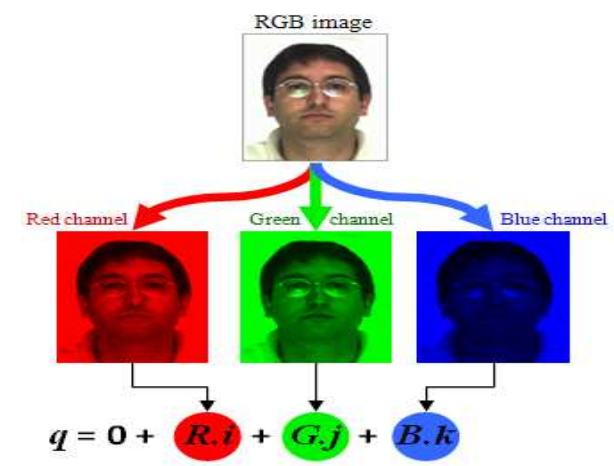

Figure 4. Quaternion representation of a color face image.

It is worth noting that quaternions are used to compactly represent the RGB color space. Therefore, the inherent color interaction is implicitly incorporated in any subsequent processing at the image level.

\subsection{Quaternion Principal Component Analysis (Q-PCA)}

The eigenvalues and eigenvectors of real matrix can be easily computed. However, computing those of a quaternion matrix is a difficult task [13]. In order to calculate the PCA projection matrix based on a quaternion representation, it is necessary to obtain the orthogonal eigenvector set of the covariance. The orthogonal eigenvectors of the PCA covariance matrix can be obtained using three PCA-based methods:

1. Quaternion Singular Value Decomposition (Q-SVD).

2. Quaternion Eigen-Value Decomposition (Q-EVD).

3. Quaternion Karhunen-Loeve Transform (Q-KLT).

The Q-SVD can be considered as a generalization of the real or complex SVD. It inherits similar properties [11]. The decomposition of a quaternion matrix by means of classical complex algorithms is based on the SVD computation of the complex adjoint matrix (CAM). Therefore, in the Q-SVD solution, a quaternion matrix can be decomposed into its singular value form by converting it into complex matrix representation.
Each quaternion matrix $\mathbb{Q}$ of size $M x N$ has an equivalent complex matrix $\mathrm{C}$ of size $2 M \gg 2 N \quad[11,10]$. Hence, the existing complex SVD algorithm can be applied to this equivalent complex matrix $\mathbb{C}$ to obtain the eigenvectors and eigenvalues of the corresponding quaternion matrix $\mathbb{Q}$.

\section{Proposed Q-PCA Algorithm}

In this paper, we propose a novel color face recognition algorithm based on quaternion PCA called Q-PCA. The proposed Q-PCA algorithm is carried out using the following procedure:

1. For each color face image, $t_{q}$, in the training data, convert $t_{q}$ into a pure quaternionic representation:

$$
t_{q}=0+R \cdot i+G \cdot j+B \cdot k
$$

2. Organize the data as an $(m \gg n)$ design matrix, $A_{q}$, where $m$ is the number of measurement types (also rows) and $n$ is the number of samples (also columns). Each matrix cell $m_{x} n_{y}$ is a quaternion number. Hence, each column $n_{y}$ represents a single vector or orthogonal representation of quaternion image pixels.

3. Normalize each measurement type, $m_{x}$, by subtracting the mean, $\mu_{q}$, and dividing by the variance, $\sigma_{q}^{2}$, to obtain $n$ orthonormal vectors of the design matrix $A_{q}$.

4. Calculate the Q-SVD or the quaternion eigenvectors, $U_{q}$, of the covariance matrix as follows:

$$
A_{q}=U_{q} \cdot \sum \cdot V_{q}^{H}
$$

where ${ }^{H}$ denotes the Hermitian transpose, $U_{q}$ and $V_{q}$ are unitary quaternion matrices $\left(U_{q} \in \mathbb{Q}_{n \times n}\right.$ and $V_{q} \in$ $\mathbb{Q}_{n \times n}$ ) such that:

$$
U_{q}^{H} \cdot Q_{q} \cdot V_{q}=\left(\begin{array}{cc}
\sum_{r} & 0 \\
0 & 0
\end{array}\right)_{n \times n}
$$

5. To recognize or match a query color face image, $f_{q}$, the quaternion form of $f_{q}$ is normalized and projected onto the eigen-face space using:

$$
P_{q}=U_{q}^{H} \cdot\left(\frac{f_{q}-\mu_{q}}{\sigma_{q}^{2}}\right)
$$

where $P_{q}$ is the projection of the image $f_{q}$.

\section{Methodology and Computer Experiments}

\subsection{Color Face Database}

The FERET database is a well known standard facial database in both color and grayscale representations provided by the US 
National Institute of Standards and Technology (NIST). It has been designed to advance the state of the art face recognition research. The database was collected at various angles in 15 sessions between August 1993 and July 1996. It contains 1564 sets of images for a total of 14126 images that includes 1199 individuals and 365 duplicate sets of images. A duplicate set is a second set of images of a person already in the database and was usually taken on a different day. For some individuals, two years have elapsed between their first and last sittings, with some subjects being photographed multiple times. This period of time was important to enable researchers to study the effects of changes in a subject's appearance that occur over years. Thus, the FERET database introduces variability by the inclusion of images taken at different dates and locations. This results in changes in lighting, scale and background. The whole database is divided into a development set, provided to researchers, and a set of sequestered images for testing. The images in the development set are used as a representative of the sequestered images. However, the available database for researchers is a subset of the database consisting of 11338 images from the total of 14126 . These images represent 994 individuals from the total of 1199 individuals. The remaining individuals and images were not provided for the researchers. The database, provided in color PPM-format, represents images in three different resolutions ranging from quarter-size $(192 \times 128$ pixels) to full-size $(768 \times 512$ pixels $)$ resolutions. There are 13 different poses in the collected database representing the subject face at various angles.

\subsection{Performance Evaluation}

A biometric evaluation protocol determines how to test a system, select the data, and measure the performance. In order to apply validation and verification to the proposed approach, the FERET evaluation protocol is used [1]. This biometric evaluation protocol is used to evaluate the performance of the proposed Q-PCA-based algorithm against typical PCA-based algorithms (gray-PCA and Avg-PCA). While the gray-PCA algorithm is based on the gray-level version of the color database images, the Avg-PCA is applied on the average face images estimated as the average of the color component images (Red, Green and Blue, respectively). A number of stress test experiments are performed on these various FR algorithms to evaluate and compare their performance using the same data sets.

\subsection{Standard Testing Subsets}

In this experimental work, FERET standard testing subsets are used to carry out the performance and statistical evaluation. Particularly, the gallery and probe sets used in FERET tests (September 1996) are selected for this purpose. Therefore, the identification scores are carried out for four categories of probes and all the tests for these probes are using a single gallery containing 993 images as indicated in Table 1 .

\begin{tabular}{|c|c|c|}
\hline Evaluation Task & Gallery Name & Probe Set \\
\hline \hline Facial Expression & FB & probe_fafb_*.names (993) \\
Low aging & duplicate I & probe_dup_1_*.names (736) \\
Illumination & fc & probe_fafc_*.names (98) \\
High aging & duplicate II & probe_dup_2_*.names (228) \\
\hline
\end{tabular}

Table 1. Gallery and four probe sets.

\begin{tabular}{|c|c|c|c|}
\hline Algorithm & Q-PCA & Gray-PCA & Avg-PCA \\
\hline \hline $\mathrm{FB}_{192 \times 128}$ & $\mathbf{0 . 6 4 6}$ & 0.624 & 0.619 \\
\hline $\mathrm{FB}_{384 \times 256}$ & $\mathbf{0 . 6 4 8}$ & 0.626 & 0.620 \\
\hline Duplicate $\mathrm{I}_{192 \times 128}$ & $\mathbf{0 . 1 4 5}$ & 0.135 & 0.133 \\
\hline Duplicate $\mathrm{I}_{384 \times 256}$ & $\mathbf{0 . 1 4 7}$ & 0.132 & 0.135 \\
\hline $\mathrm{fc}_{192 \times 128}$ & 0.010 & 0.010 & 0.010 \\
\hline fc $_{384 \times 256}$ & 0.010 & 0.010 & 0.010 \\
\hline Duplicate $\mathrm{II}_{192 \times 128}$ & 0.004 & 0.004 & 0.004 \\
\hline Duplicate $\mathrm{II}_{384 \times 256}$ & 0.004 & 0.004 & 0.004 \\
\hline \hline
\end{tabular}

Table 2. Match scores of the top-rank score.

\subsection{Performance Results}

Table 2 gives the fraction of probes whose gallery match is top ranked (i.e., the rank equals to 1 ). Hence, each number in this table represents the correct total match score considering the match with only one nearest-neighbor to each tested sample. This is effectively the performance on forced identification (i.e., best guess). As shown in the table, each probe category has two score numbers representing scores of two different resolutions. Table 3 gives the estimated average score of the total match score against each probes (i.e., the average of scores located along a rank from 1 to 50) which consider the average of the first 50 identification estimations. Hence, each number in this table represents the average of correct total match scores of the ranks from 1 to 50 (i.e., from 1 to 50 nearest-neighbors) for each test sample. This is effectively the total performance on forced identification (i.e., best $1,2, \ldots, 50$ guesses). In order to evaluate and compare the performance of different FR algorithms, two aspects are taken in account. The first is the evaluation of an algorithm based on the achieved top-rank match score as reported in Table 2. The second is the total-average of total match scores that depends on averaging the total of scores achieved as listed in Table 3. So, according to the scoring results summarized in Tables 2-3, the Q-PCA algorithm yielded the highest top-rank match score, and achieved the best performance in the identification task. The superior performance of the proposed algorithm versus its PCA-based counterpart is also highlighted in Figure 5 which illustrates the matching performance in terms of receiver operating characteristics (ROC) curve.

\section{Conclusions}

In this paper, we have proposed a new algorithm for color face recognition. The proposed algorithm is based on the quaternionic principal component analysis (Q-PCA). Unlike exist- 


\begin{tabular}{|c|c|c|c|}
\hline Algorithm & Q-PCA & Gray-PCA & Avg-PCA \\
\hline \hline $\mathrm{FB}_{192 \times 128}$ & $\mathbf{0 . 8 8 0}$ & 0.867 & 0.865 \\
\hline $\mathrm{FB}_{384 \times 256}$ & $\mathbf{0 . 8 8 1}$ & 0.868 & 0.865 \\
\hline Duplicate $\mathrm{I}_{192 \times 128}$ & $\mathbf{0 . 2 8 4}$ & 0.277 & 0.277 \\
\hline Duplicate $\mathrm{I}_{384 \times 256}$ & $\mathbf{0 . 2 8 5}$ & 0.277 & 0.278 \\
\hline fc $_{192 \times 128}$ & $\mathbf{0 . 0 4 2}$ & 0.037 & 0.041 \\
\hline fc $_{384 \times 256}$ & $\mathbf{0 . 0 4 1}$ & 0.037 & 0.041 \\
\hline Duplicate $\mathrm{II}_{192 \times 128}$ & $\mathbf{0 . 0 5 2}$ & 0.047 & 0.050 \\
\hline Duplicate $\mathrm{II}_{384 \times 256}$ & $\mathbf{0 . 0 5 2}$ & 0.047 & 0.050 \\
\hline \hline
\end{tabular}

Table 3. Average score of the total match scores of rank 50.

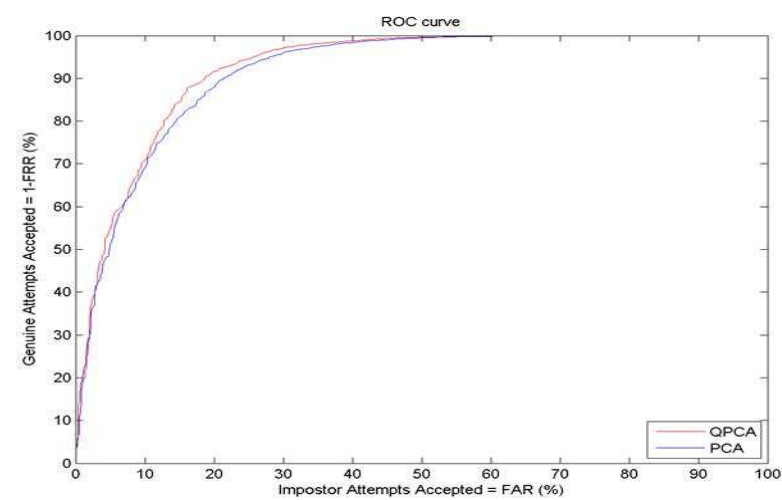

Figure 5. ROC performance of Q-PCA-based versus-based PCA algorithms.

ing color-based algorithms, the proposed one enables the implicit use of the rich color interaction that exists between the color components in facial images. The FERET testing scenario used in the performance evaluation of the standard FR algorithms clearly indicates that the proposed QPCA-based algorithm yields the best performance in both identification and verification modes.

\section{Acknowledgements}

E. S. Jaha would like to thank King Abdul-Aziz University for supporting his work and L. Ghouti acknowledges the support of King Fahd University of Petroleum and Minerals to carry out this research.

\section{References}

[1] P. J. Phillips, M. Hyeonjoon, S. A. Rizvi and P. J. Rauss, "The FERET evaluation methodology for face-recognition algorithms," IEEE Trans. on Pattern Analysis and Machine Intelligence, vol. 22, no.10, pp.1090-1104, Oct. 2000.

[2] J. Yang and C. Liu, "A General Discriminant Model for Color Face Recognition," IEEE 11th International Conference on Computer Vision (ICCV 2007), pp. 1-6, Oct. 2007.
[3] P. Shih and C. Liu, "Extracting Efficient Color Features for Face Recognition Using Evolutionary Computation," 6th International Conference on Computational Intelligence and Multimedia Applications, pp. 285-290, Aug. 2005.

[4] J. Choi, Y. Ro and K. Plataniotis, "Color Face Recognition for Degraded Face Images," IEEE Transactions on Systems, Man, and Cybernetics, Part B: Cybernetics, vol. 39, no. 5, pp. 1217-1230, Oct. 2009.

[5] C. Wang and X. Bai, "Color Face Recognition Based on Revised NMF Algorithm," Second International Conference on Future Information Technology and Management Engineering (FITME '09), pp. 455-458, Dec. 2009.

[6] M. Thomas, C. Kambhamettu and S. Kumar, "Face Recognition Using a Color Subspace LDA Approach," 20th IEEE International Conference on Tools with Artificial Intelligence (ICTAI '08), vol. 1, pp. 231-235, Nov. 2008.

[7] C. Wang, B. Yin, X. Bai and Y. Sun, "Color Face Recognition Based on 2DPCA," 19th International Conference on Pattern Recognition (ICPR 2008), pp. 1-4, Dec. 2008.

[8] H. Moon and P. Phillips, "Computational and Performance Aspects of PCA-based Face Recognition Algorithms," Perception, vol. 30, no. 3, pp. 301-321, 2001.

[9] N. Le Bihan and S. Sangwine, "Quaternion Principal Component Analysis of Color Images," International Conference on Image Processing Proceedings (ICIP 2003), vol. 1, pp. 809-12, Sept. 2003.

[10] S. Sangwine and N. Le Bihan, "Computing the SVD of a quaternion matrix," 7th IMA Conference on Mathematics in Signal Processing, 2006.

[11] L. Shi, "Exploration in Quaternion Colour," MS Thesis, Simon Fraser University, Canada, 2005.

[12] L. Ding and H. Feng, "Quaternion K-L Transform and Biomimetic Pattern Recognition Approaches for Colorface Recognition," IEEE International Conference on Intelligent Computing and Intelligent Systems (ICIS 2009), vol. 1, pp. 165-169, Nov. 2009.

[13] S. Chen, Y. Sun and B. Yin, "A Novel Hybrid Approach Based on Sub-pattern Technique and Extended 2DPCA for Color Face Recognition," 11th IEEE International Symposium on Multimedia (ISM '09), pp. 630-634, Dec. 2009.

[14] L. Ghouti and F. S. Al-Qunaieer, "Color Iris Recognition Using Quaternion Phase Correlation,” ECSIS Symposium on Bio-inspired, Learning, and Intelligent Systems for Security, pp. 20-25, July 2009. 